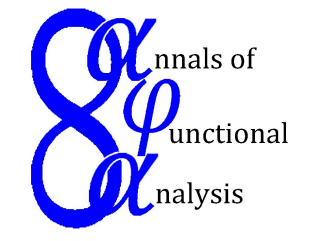

Ann. Funct. Anal. 5 (2014), no. 1, 56-62

$\mathscr{A}$ NNALS OF $\mathscr{F}$ UnCTIONAL $\mathscr{A}$ NALYSIS

ISSN: 2008-8752 (electronic)

URL:www.emis.de/journals/AFA/

\title{
ESTIMATES FOR THE NUMERICAL RADIUS AND THE SPECTRAL RADIUS OF THE FROBENIUS COMPANION MATRIX AND BOUNDS FOR THE ZEROS OF POLYNOMIALS
}

\author{
AMER ABU-OMAR ${ }^{1}$ AND FUAD KITTANEH ${ }^{2 *}$ \\ Dedicated to Professor Tsuyoshi Ando in celebration of his distinguished achievements in \\ Matrix Analysis and Operator Theory \\ Communicated by M. S. Moslehian
}

ABstract. We apply numerical radius and spectral radius estimates to the Frobenius companion matrices of monic polynomials to derive new bounds for their zeros and give different proofs of some known bounds.

\section{INTRODUCTION}

Let $M_{n}(\mathbb{C})$ denote the algebra of all $n \times n$ complex matrices. For $A \in M_{n}(\mathbb{C})$, let $r(A), w(A)$, and $\|A\|$ denote the spectral radius, the numerical radius, and the spectral norm of $A$, respectively. Recall that $w(A)=\max _{\|x\|=1}|\langle A x, x\rangle|$. It is well-known that $w(\cdot)$ defines a norm on $M_{n}(\mathbb{C})$ and

$$
r(A) \leq w(A) \leq\|A\|
$$

with equality if $A$ is normal.

Let $p(z)=z^{n}+a_{n} z^{n-1}+\cdots+a_{2} z+a_{1}$ be a monic polynomial of degree $n>2$, where $a_{1}, a_{2}, \ldots, a_{n}$ are complex numbers with $a_{1} \neq 0$. Then the Frobenius

Date: Received: 23 July 2013; Accepted: 9 August 2013.

* Corresponding author.

2010 Mathematics Subject Classification. Primary 47A12; Secondary 15A60, 26C10, 30C15.

Key words and phrases. Frobenius companion matrix, zeros of polynomials, numerical radius, spectral radius, inequality. 
companion matrix of $p$ is given by

$$
C(p)=\left[\begin{array}{ccccc}
-a_{n} & -a_{n-1} & \cdots & -a_{2} & -a_{1} \\
1 & 0 & \cdots & 0 & 0 \\
0 & 1 & \cdots & 0 & 0 \\
\vdots & \vdots & \ddots & \vdots & \vdots \\
0 & 0 & \cdots & 1 & 0
\end{array}\right] .
$$

It is well-known that the zeros of $p$ coincide with the eigenvalues of $C(p)$ (see, e.g. [7, p. 316]). Hence, if $z$ is any zero of $p$, then

$$
|z| \leq r(C(p)) \leq w(C(p)) .
$$

In this paper, we obtain estimates for the numerical radius and the spectral radius of the Frobenius companion matrix and derive new bounds for the zeros of polynomials. Also, we give different proofs of some known bounds of Fujii and Kubo, Abdurakhmanov, and Kittaneh. Other related bounds can be found in $[10,11]$, and references therein.

\section{Main Results}

In order to achieve our goal, we need the following lemmas. The first two lemmas are well-known and they can be found in [13] and [6, pp. 8-9], respectively.

Lemma 2.1. Let $A \in M_{n}(\mathbb{C})$. Then

$$
w(A)=\max _{\theta \in \mathbb{R}}\left\|\operatorname{Re}\left(e^{i \theta} A\right)\right\| .
$$

Applying the above relation to the block matrix $\left[\begin{array}{cc}0 & B \\ C & 0\end{array}\right]$, where $B \in M_{k \times m}(\mathbb{C})$ and $C \in M_{m \times k}(\mathbb{C})$, we have

$$
w\left(\left[\begin{array}{cc}
0 & B \\
C & 0
\end{array}\right]\right)=\frac{1}{2} \max _{\theta \in \mathbb{R}}\left\|e^{i \theta} B+e^{-i \theta} C^{*}\right\|,
$$

and so

$$
w\left(\left[\begin{array}{cc}
0 & B \\
C & 0
\end{array}\right]\right) \leq \frac{1}{2}(\|B\|+\|C\|)
$$

Here $M_{k \times m}(\mathbb{C})$ is the space of all $k \times m$ complex matrices.

Lemma 2.2. Let $L_{n}$ be the $n \times n$ matrix given by

$$
L_{n}=\left[\begin{array}{ccccc}
0 & 0 & \cdots & 0 & 0 \\
1 & 0 & \cdots & 0 & 0 \\
0 & 1 & \cdots & 0 & 0 \\
\vdots & \vdots & \ddots & \vdots & \vdots \\
0 & 0 & \cdots & 1 & 0
\end{array}\right]
$$

Then $w\left(L_{n}\right)=\cos \frac{\pi}{n+1}$.

The third lemma contains numerical radius inequalities for general $2 \times 2$ block matrices. These inequalities are special cases of more general ones which have been recently given in [3] for Hilbert space operators. 
Lemma 2.3. Let $A \in M_{k}(\mathbb{C}), B \in M_{k \times m}(\mathbb{C}), C \in M_{m \times k}(\mathbb{C})$, and $D \in M_{m}(\mathbb{C})$, and let $\mathbf{T}=\left[\begin{array}{ll}A & B \\ C & D\end{array}\right]$. Then

$$
\begin{aligned}
w(\mathbf{T}) & \leq w\left(\left[\begin{array}{cc}
w(A) & w\left(\mathbf{T}_{O}\right) \\
w\left(\mathbf{T}_{O}\right) & w(D)
\end{array}\right]\right) \\
& =\frac{1}{2}\left(w(A)+w(D)+\sqrt{(w(A)-w(D))^{2}+4 w^{2}\left(\mathbf{T}_{O}\right)}\right),
\end{aligned}
$$

where $\mathbf{T}_{O}=\left[\begin{array}{ll}0 & B \\ C & 0\end{array}\right]$.

Remark 2.4. It follows from the inequality (2.2) that

$$
\begin{aligned}
& \frac{1}{2}\left(w(A)+w(D)+\sqrt{(w(A)-w(D))^{2}+4 w^{2}\left(\mathbf{T}_{O}\right)}\right) \\
\leq & \frac{1}{2}\left(w(A)+w(D)+\sqrt{(w(A)-w(D))^{2}+(\|B\|+\|C\|)^{2}}\right),
\end{aligned}
$$

and so by Lemma 2.3,

$$
\begin{aligned}
w(\mathbf{T}) & \leq w\left(\left[\begin{array}{cc}
w(A) & \|B\| \\
\|C\| & w(D)
\end{array}\right]\right) \\
& =\frac{1}{2}\left(w(A)+w(D)+\sqrt{(w(A)-w(D))^{2}+(\|B\|+\|C\|)^{2}}\right) .
\end{aligned}
$$

It should be mentioned here that the weaker inequality (2.5) has been obtained in [12]. This inequality, in turn, is sharper than an earlier inequality of Hou and $\mathrm{Du}[8]$, which says that

$$
\begin{aligned}
w(\mathbf{T}) & \leq w\left(\left[\begin{array}{ll}
\|A\| & \|B\| \\
\|C\| & \|D\|
\end{array}\right]\right) \\
& =\frac{1}{2}\left(\|A\|+\|D\|+\sqrt{(\|A\|-\|D\|)^{2}+(\|B\|+\|C\|)^{2}}\right) .
\end{aligned}
$$

The inequality (2.5) has been recently employed in [2] to establish a general spectral radius inequality, which yields spectral radius inequalities for sums, products, and commutators of operators. In particular, it has been shown that if $A$ and $B$ are bounded linear operators acting on a Hilbert space, then

$$
r(A+B) \leq \frac{1}{2}\left(w(A)+w(B)+\sqrt{(w(A)-w(B))^{2}+4 \min \{\|A B\|,\|B A\|\}}\right) .
$$

Now, we are in a position to give our new proofs of the following known bounds.

Theorem 2.5 (Fujii and Kubo [5]). If $z$ is any zero of $p$, then

$$
|z| \leq \frac{1}{2}\left(\left|a_{n}\right|+\sqrt{\sum_{j=1}^{n}\left|a_{j}\right|^{2}}\right)+\cos \frac{\pi}{n+1} .
$$


Proof. Let $v$ denote the row vector $\left[\begin{array}{lllll}-a_{n-1} & -a_{n-2} & \cdots & -a_{2} & -a_{1}\end{array}\right]$, and let $A$ and $B$ denote the $n \times n$ matrices $\left[\begin{array}{cc}-a_{n} & v \\ 0 & 0\end{array}\right]$ and $L_{n}$, respectively. Applying the triangle inequality, and using the inequality (2.5) together with Lemma 2.2, we have

$$
w(C(p))=w(A+B) \leq w(A)+w\left(L_{n}\right) \leq \frac{1}{2}\left(\left|a_{n}\right|+\sqrt{\sum_{j=1}^{n}\left|a_{j}\right|^{2}}\right)+\cos \frac{\pi}{n+1},
$$

from which the inequality (2.7) follows.

Theorem 2.6 (Abdurakhmanov [1]). If $z$ is any zero of $p$, then

$$
|z| \leq \frac{1}{2}\left(\left|a_{n}\right|+\cos \frac{\pi}{n}+\sqrt{\left(\left|a_{n}\right|-\cos \frac{\pi}{n}\right)^{2}+\left(1+\sqrt{\sum_{j=1}^{n-1}\left|a_{j}\right|^{2}}\right)^{2}}\right)
$$

Proof. Let $e_{1}$ denote the $(n-1)$ column vector $\left[\begin{array}{lllll}1 & 0 & \cdots & 0 & 0\end{array}\right]^{t}$. Then $C(p)=$ $\left[\begin{array}{cc}-a_{n} & v \\ e_{1} & L_{n-1}\end{array}\right]$. The inequality (2.8) follows directly by applying the inequality (2.5) to the matrix $C(p)$ (partitioned as above) and recalling that $|z| \leq w(C(p))$.

Theorem 2.7 (Kittaneh [9]). If $z$ is any zero of $p$, then

$$
|z| \leq \frac{1}{2}\left(\left|a_{n}\right|+\cos \frac{\pi}{n}+\sqrt{\left(\left|a_{n}\right|-\cos \frac{\pi}{n}\right)^{2}+\left(\left|a_{n-1}\right|+1\right)^{2}+\sum_{j=1}^{n-2}\left|a_{j}\right|^{2}}\right) .
$$

Proof. Let $\mathbf{T}_{O}=\left[\begin{array}{cc}0 & v \\ e_{1} & 0\end{array}\right]$. Then by Lemma 2.1,

$$
w\left(\mathbf{T}_{O}\right)=\frac{1}{2} \max _{\theta \in \mathbb{R}}\left\|\left[\begin{array}{cc}
0 & u \\
u^{*} & 0
\end{array}\right]\right\|,
$$

where $u=\left[\begin{array}{lllll}-e^{i \theta} a_{n-1}+e^{-i \theta} & -e^{i \theta} a_{n-2} & \cdots & -e^{i \theta} a_{2} & -e^{i \theta} a_{1}\end{array}\right]$. Thus,

$$
\begin{aligned}
w\left(\mathbf{T}_{O}\right) & =\frac{1}{2} \max _{\theta \in \mathbb{R}}\|u\|=\frac{1}{2} \max _{\theta \in \mathbb{R}} \sqrt{\left|e^{i \theta} a_{n-1}-e^{-i \theta}\right|^{2}+\sum_{j=1}^{n-2}\left|a_{j}\right|^{2}} \\
& =\frac{1}{2} \sqrt{\left(\left|a_{n-1}\right|+1\right)^{2}+\sum_{j=1}^{n-2}\left|a_{j}\right|^{2}} .
\end{aligned}
$$

Now, by using the relation (2.10), the inequality (2.9) follows by applying Lemma 2.3 to the matrix $C(p)=\left[\begin{array}{cc}-a_{n} & v \\ e_{1} & L_{n-1}\end{array}\right]$ and recalling that $|z| \leq w(C(p))$. 
Remark 2.8. Using the inequality (2.4), it is easily seen from our proofs of Theorem 2.6 and Theorem 2.7 that the inequality (2.9) is sharper than the inequality (2.8).

In [4], Buzano obtained the following extension of Schwarz's inequality: If $a, b, x$ are vectors in an inner product space, then

$$
|\langle a, x\rangle||\langle x, b\rangle| \leq \frac{\|a\|\|b\|+|\langle a, b\rangle|}{2}\|x\|^{2} .
$$

A simple proof of this inequality has been given in [5]. Employing Buzano's inequality, we prove the following lemma.

Lemma 2.9. Let $A, B \in M_{n}(\mathbb{C})$. Then

$$
w(A+B) \leq \sqrt{w^{2}(A)+w^{2}(B)+\|A\|\|B\|+w\left(B^{*} A\right)}
$$

Proof. For any unit vector $x \in \mathbb{C}^{n}$, we have

$$
\begin{aligned}
|\langle(A+B) x, x\rangle|^{2} & \leq(|\langle A x, x\rangle|+|\langle B x, x\rangle|)^{2} \\
& =|\langle A x, x\rangle|^{2}+|\langle B x, x\rangle|^{2}+2|\langle A x, x\rangle||\langle B x, x\rangle| \\
& =|\langle A x, x\rangle|^{2}+|\langle B x, x\rangle|^{2}+2|\langle A x, x\rangle||\langle x, B x\rangle| \\
& \leq|\langle A x, x\rangle|^{2}+|\langle B x, x\rangle|^{2}+\|A x\|\|B x\|+|\langle A x, B x\rangle| \\
& =|\langle A x, x\rangle|^{2}+|\langle B x, x\rangle|^{2}+\|A x\|\|B x\|+\left|\left\langle B^{*} A x, x\right\rangle\right| \\
& \leq w^{2}(A)+w^{2}(B)+\|A\|\|B\|+w\left(B^{*} A\right) .
\end{aligned}
$$

Thus,

$$
w^{2}(A+B)=\max _{\|x\|=1}|\langle(A+B) x, x\rangle|^{2} \leq w^{2}(A)+w^{2}(B)+\|A\|\|B\|+w\left(B^{*} A\right),
$$

and so

$$
w(A+B) \leq \sqrt{w^{2}(A)+w^{2}(B)+\|A\|\|B\|+w\left(B^{*} A\right)}
$$

as required.

It should be mentioned here that Lemma 2.9 can be extended in a natural way to Hilbert space operators.

Using Lemma 2.9, we derive the following new bound.

Theorem 2.10. If $z$ is any zero of $p$, then

$$
|z| \leq w(C(p)) \leq \sqrt{\frac{1}{4}\left(\left|a_{n}\right|+\alpha\right)^{2}+\alpha+\cos ^{2} \frac{\pi}{n+1}},
$$

where $\alpha=\sqrt{\sum_{j=1}^{n}\left|a_{j}\right|^{2}}$.

Proof. Let $A$ and $B$ be as in the proof of Theorem 2.5. Then the second inequality in (2.11) follows by applying Lemma 2.9 to the matrix $C(p)$ written as $C(p)=$ $A+B$, and noting that $B^{*} A=0$.

In the rest of the paper, we use the inequality (2.6) to give an estimate for the spectral radius of the Frobenius companion matrix $C(p)$ and derive a new bound for the zeros of $p$. 
Theorem 2.11. If $z$ is any zero of $p$, then

$$
|z| \leq r(C(p)) \leq \frac{1}{2}\left(\frac{1}{2}\left(\left|a_{n}\right|+\alpha\right)+\cos \frac{\pi}{n+1}+\sqrt{\left(\frac{1}{2}\left(\left|a_{n}\right|+\alpha\right)-\cos \frac{\pi}{n+1}\right)^{2}+4 \alpha^{\prime}}\right)
$$

where $\alpha^{\prime}=\sqrt{\sum_{j=1}^{n-1}\left|a_{j}\right|^{2}}$.

Proof. Let $A$ and $B$ be as in the proof of Theorem 2.5. Then the second inequality in (2.12) follows by applying the inequality (2.6) to the matrix $C(p)=A+B$, and noting that $\|A B\|=\alpha^{\prime}$ and $\|B A\|=\alpha$.

Consider the polynomial $p(z)=z^{3}+2 z^{2}+1$. Simple calculations show that the Fujii-Kubo bound in Theorem 2.5 is not uniformly sharper than our new bounds given in Theorem 2.10 and Theorem 2.11. In fact, if $b_{F K}=\frac{1}{2}\left(\left|a_{n}\right|+\alpha\right)+\cos \frac{\pi}{n+1}$, $b_{1}=\sqrt{\frac{1}{4}\left(\left|a_{n}\right|+\alpha\right)^{2}+\alpha+\cos ^{2} \frac{\pi}{n+1}}$, and

$$
b_{2}=\frac{1}{2}\left(\frac{1}{2}\left(\left|a_{n}\right|+\alpha\right)+\cos \frac{\pi}{n+1}+\sqrt{\left(\frac{1}{2}\left(\left|a_{n}\right|+\alpha\right)-\cos \frac{\pi}{n+1}\right)^{2}+4 \alpha^{\prime}}\right),
$$

then it is easy to show that $b_{1}<b_{F K}$ if and only if $\frac{\alpha}{\left|a_{n}\right|+\alpha}<\cos \frac{\pi}{n+1}$ and $b_{2}<b_{F K}$ if and only if $\frac{2 \alpha^{\prime}}{\left|a_{n}\right|+\alpha}<\cos \frac{\pi}{n+1}$.

\section{REFERENCES}

1. A.A. Abdurakhmanov, Geometry of a Hausdorff domain in problems of localization of the spectrum of arbitrary matrices, Math. USSR Sb. 59 (1988), 39-51.

2. A. Abu-Omar and F. Kittaneh, A numerical radius inequality involving the generalized Aluthge transform, Studia Math. 216 (2013), 69-75.

3. A. Abu-Omar and F. Kittaneh, Numerical radius inequalities for $n \times n$ operator matrices, Linear Algebra Appl. (to appear).

4. M.L. Buzano, Generalizzatione della diseguaglianza di Cauchy-Schwarz (Italian), Rend. Sem. Mat. Univ. e Politech. Torino 31 (1971/73), 405-409 (1974).

5. M. Fujii and F. Kubo, Buzano's inequality and bounds for roots of algebraic equations, Proc. Amer. Math. Soc. 117 (1993), 359-361.

6. K.E. Gustafson and D.K.M. Rao, Numerical Range, Springer, New York, 1997.

7. R.A. Horn and C.R. Johnson, Matrix Analysis, Cambridge University Press, Cambridge, 1985.

8. J.C. Hou and H.K. Du, Norm inequalities of positive operator matrices, Integral Equations Operator Theory 22 (1995), 281-294.

9. F. Kittaneh, Bounds for the zeros of polynomials from matrix inequalities, Arch. Math. (Basel) 81 (2003), 601-608.

10. F. Kittaneh, A numerical radius inequality and an estimate for the numerical radius of the Frobenius companion matrix, Studia Math. 158 (2003), 11-17.

11. F. Kittaneh and K. Shebrawi, Bounds and majorization relations for the zeros of polynomials, Numer. Funct. Anal. Optim. 30 (2009), 98-110.

12. K. Paul and S. Bag, On numerical radius of a matrix and estimation of bounds for zeros of a polynomial, Int. J. Math. Math. Sci. (2012), Art. ID 129132, 15 pp. 
13. T. Yamazaki, On upper and lower bounds of the numerical radius and an equality condition, Studia Math. 178 (2007), 83-89.

1 Department of Basic Sciences and Mathematics, Philadelphia University, AMMAN, JORDAN.

E-mail address: aabuomar@philadelphia.edu.jo

${ }^{2}$ Department of Mathematics, The University of Jordan, Amman, Jordan.

E-mail address: fkitt@ju.edu.jo 